\title{
A New Learning Controller for Periodic Disturbance Rejection
}

\author{
Sanem Evren Han and Mustafa Unel*
}

\begin{abstract}
A new acceleration based learning control approach is developed to tackle the robust periodic trajectory tracking problem for robot manipulators. The acceleration feedback is incorporated into the learning feedforward term to provide high stiffness to the system against unknown periodic dynamics with a known period. A cascaded high gain observer is used to obtain reliable position, velocity and acceleration signals from noisy encoder measurements. A closed-loop stability proof is provided where it is shown that all system signals remain bounded and the proposed learning controller achieves global asymptotic position tracking. Simulation results obtained from a high fidelity model show that the proposed controller outperforms the learning controller that does not utilize the acceleration feedback.
\end{abstract}

\section{INTRODUCTION}

Learning controllers have gained remarkable importance for repetitive robot tasks. The aim of a learning control is to achieve desired system performance by updating the control input from past errors either repeatedly over a fixed finite time interval, or repetitively (cyclically) over an infinite time interval. Typical learning control strategies are generally categorized into iterative learning control (ILC) and repetitive control (RC). ILC [1] provides satisfactory tracking repeatedly on a fixed time (pre-specified) interval whereas RC [2] handles the problem of the periodic reference tracking and disturbance rejection.

ILC is an iteration-domain method and requires to reset the system to the initial condition at the beginning of each cycle to perform the repetitive tasks. Repetitive control, on the other hand, is a time-domain technique. RC needs no initial repositioning and the system operates continuously. ILCs have been applied for trajectory tracking of different robotic systems [3]-[4].

In parallel to developments in those controllers, repetitive controllers have attracted many researchers to solve similar tracking problems without the requirement for initial repositioning. Earlier works of the repetitive controllers have been developed by [2] for linear time-invariant systems. The stability analysis was conducted for linear processes that repeat continuously [5]. RC has been applied on nonlinear systems [6]-[8]. Messner et al. [6] identified and compensated a nonlinear disturbance function where it is represented as an integral of a predefined a kernel function multiplied by an unknown influence function. Using the past information of the plant, the learning rule was utilized to indirectly estimate

*Corresponding Author

Sanem Evren Han and Mustafa Unel are with Faculty of Engineering and Natural Sciences, Sabanci University, Istanbul, Turkey (sanemevren, munel) @sabanciuniv.edu the disturbance function by updating the influence function estimate. This controller achieves asymptotic disturbance cancellation. Dixon et al. [7] and Cao et al. [8] proposed repetitive learning controllers for nonlinear systems with exogenous periodic disturbances that satisfy the matching condition. Similar to ILC, the repetitive controller has been widely applied to eliminate periodic disturbances in engineering applications [9]-[11].

In this paper, a new learning controller is developed by incorporating acceleration feedback into the standard learning control feedforward term to achieve a global position tracking for n-rigid link robotic manipulators despite unknown periodic dynamics. The proposed acceleration based learning controller should improve the robustness of the system against unknown periodic dynamics because the effects of those disturbances can manifest themselves in the acceleration signals first. Reliable position, velocity and acceleration feedback signals are required for a satisfactory trajectory tracking performance. However, it is difficult to obtain velocity and acceleration signals from noisy encoder measurements due to sensor quantization errors. A cascaded high gain observer (CHGO) is designed to estimate reliable position, velocity and acceleration signals. Estimated position, velocity and acceleration signals are used in the acceleration based learning controller that is developed on a n-rigid link robotic manipulator. The filtered error is modified to include the integral of the position tracking error. A closed-loop stability proof is provided which shows that all system signals are bounded and global asymptotic position tracking is guaranteed. Results that are obtained from a high fidelity simulation model demonstrates the validity and effectiveness of the developed learning controller.

The remainder of this paper is organized as follows: Section II presents dynamical model of a n-rigid link robotic manipulator and a 2 DOF pan-tilt system. Section III develops a cascaded high gain observer (CHGO) to estimate reliable position, velocity and acceleration signals. In Section IV, a new acceleration based learning controller is designed and the closed-loop stability proof is presented. Section V provides simulation results where the effectiveness of the proposed control approach is validated on a 2 DOF pan-tilt robot. Finally, Section VI concludes the paper with some important remarks.

\section{Modeling}

The nonlinear dynamical model of a n-rigid link robotic manipulator is formulated as follows [12]: 


$$
M(q) \ddot{q}+C(q, \dot{q}) \dot{q}+F_{\nu} \dot{q}+F_{s} \operatorname{sgn}(\dot{q})+G(q)=\tau
$$

where $q(t), \dot{q}(t), \ddot{q}(t) \in \mathbb{R}^{n}$ are the link positions, velocities and accelerations, $M(q) \in \mathbb{R}^{n \times n}$ denotes the symmetric, positive-definite inertia matrix, $C(q, \dot{q}) \in \mathbb{R}^{n \times n}$ represents centripetal-Coriolis matrix, $G(q) \in \mathbb{R}^{n}$ is the gravity vector, $F_{v}$ and $F_{s} \in \mathbb{R}^{n \times n}$ are constant, diagonal, positive-definite, viscous and static friction coefficient matrices, $\operatorname{sgn}(\dot{q})$ is the signum function applied to the link velocities, and $\tau \in$ $\mathbb{R}^{n}$ is the torque control input. For the subsequent control development and stability analysis, the following important properties will be utilized.

\section{Property 1: Symmetric and Positive-Definite Inertia Matrix}

The symmetric and positive-definite inertia matrix, $M(q)$, satisfies the following inequality:

$$
\alpha_{1}\|\eta\|^{2} \leq \eta^{T} M(q) \eta \leq \alpha_{2}\|\eta\|^{2} \quad \forall \eta \in \mathbb{R}^{n}
$$

where $\alpha_{1}, \alpha_{2} \in \mathbb{R}$ are known positive constants, $\|\cdot\|$ denotes the standard Euclidean norm.

\section{Property 2: Skew-Symmetry}

The inertia and centripetal-Coriolis matrices satisfy the following skew-symmetric relationship:

$$
\eta^{T}\left(\frac{1}{2} \dot{M}(q)-C(q, \dot{q})\right) \eta=0 \quad \forall \eta \in \mathbb{R}^{n}
$$

where $\dot{M}$ is the time derivative of the inertia matrix.

\section{Property 3: Bounding Inequalities}

The upper bounds for the norms of the centripetal-Coriolis, gravity, and viscous friction terms can be obtained as follows:

$$
\|C(q, \dot{q})\|_{i \infty} \leq \sigma_{c 1}\|\dot{q}\|,\|G(q)\| \leq \sigma_{g},\left\|F_{v}\right\|_{i \infty} \leq \sigma_{f_{v}}
$$

where $\sigma_{c 1}, \sigma_{g}, \sigma_{f_{v}} \in \mathbb{R}$ represents known positive constants and $\|\cdot\|_{i \infty}$ is the induced infinity norm of a matrix.

Remark 1: Using the assumptions given in (2)-(4), it can be concluded that the torque control input, $\tau$, is bounded when all the terms on the left-hand side of (1) are bounded provided that $q(t), \dot{q}(t), \ddot{q}(t) \in \mathscr{L}_{\infty}$.

This section also provides a dynamical model of a 2 DOF pan-tilt manipulator in Figure 1. It will be used to evaluate the performance of the proposed control algorithm in Section $\mathrm{V}$. The nonlinear model of the pan-tilt system based on the Euler-Lagrange formulation is as follows [13]-[14]:

$$
M(q) \ddot{q}+C(q, \dot{q}) \dot{q}+G(q)+F_{v} \dot{q}+F_{s} \operatorname{sgn}(\dot{q})=\tau
$$

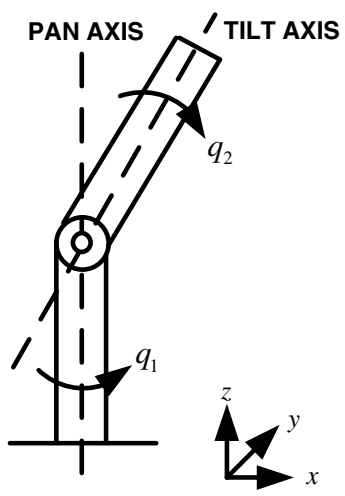

Fig. 1. Pan-tilt mechanism

where

$$
\begin{aligned}
& q=\left[\begin{array}{ll}
q_{1} & q_{2}
\end{array}\right]^{T}, \quad \tau=\left[\begin{array}{ll}
\tau_{1} & \tau_{2}
\end{array}\right]^{T} \\
& M(q)=\left[\begin{array}{ll}
M_{11} & M_{12} \\
M_{21} & M_{22}
\end{array}\right], \quad C(q, \dot{q})=\left[\begin{array}{ll}
C_{11} & C_{12} \\
C_{21} & C_{22}
\end{array}\right] \\
& G(q)=\left[\begin{array}{ll}
0 & 0.5 g m_{2} l_{2} \cos q_{2}
\end{array}\right]^{T}, \quad F_{v}(\dot{q})=\left[\begin{array}{ll}
v_{1} \dot{q}_{1} & v_{2} \dot{q}_{2}
\end{array}\right]^{T} \\
& F_{S}(\dot{q})=\left[\begin{array}{ll}
k_{1} \operatorname{sgn}\left(\dot{q}_{1}\right) \quad k_{2} \operatorname{sgn}\left(\dot{q}_{2}\right)
\end{array}\right]^{T} \\
& M_{11}=\frac{1}{2} m_{1} l_{1}^{2}+m_{2} l_{1}^{2}+m_{2} l_{1} l_{2} \cos q_{2}+\frac{1}{3} m_{2} l_{2}^{2} \cos ^{2} q_{2}+J_{1} \\
& M_{22}=\frac{1}{3} m_{2} l_{2}^{2}+J_{2}, \quad M_{12}=M_{21}=0 \\
& C_{11}=-m_{2} l_{1} l_{2} \dot{q}_{2} \sin q_{2}, \quad C_{12}=-\frac{1}{3} m_{2} l_{2}^{2} \dot{q}_{1} \sin 2 q_{2}
\end{aligned}
$$$$
C_{21}=\dot{q}_{1}\left(\frac{1}{2} m_{2} l_{1} l_{2} \sin q_{2}+\frac{1}{6} m_{2} l_{2}^{2} \sin 2 q_{2}\right), \quad C_{22}=0
$$

where $J_{1}$ and $J_{2}$ are motor inertias, $m_{1}$ and $m_{2}$ are the pan and tilt masses, $l_{1}$ is the radius, $l_{2}$ is the length, $v_{1}$ and $v_{2}$ are viscous friction coefficients, and $k_{1}$ and $k_{2}$ are static friction coefficients.

\section{ENCODER MODELING AND ESTIMATION BY A Cascaded High Gain Observer}

This section models an encoder and develops the proposed observer to estimate position, velocity and acceleration feedback signals.

\section{A. Encoder Modeling}

Encoders measure joint angles in control applications. Encoders are modeled by corrupting true sensor measurements with sensor errors as follows:

$$
q_{m}=q_{0}+b_{e}+\mu_{e}
$$

where $q_{m} \in \mathbb{R}^{n}$ is the measured encoder data, $q_{0} \in \mathbb{R}^{n}$ is the true encoder measurement, $b_{e} \in \mathbb{R}^{n}$ represents encoder biases and $\mu_{e} \in \mathbb{R}^{n}$ denotes encoder white Gaussian noises. 


\section{B. A Cascaded High Gain Observer}

A new high gain observer where two different observers are developed in a cascaded structure is proposed as depicted in Figure 2. This observer provides reliable estimates of link positions, $\hat{x}_{o_{1}}$, velocities, $\hat{z}_{o_{1}}$, and accelerations, $\hat{z}_{o_{2}}$, from noisy encoder measurements.

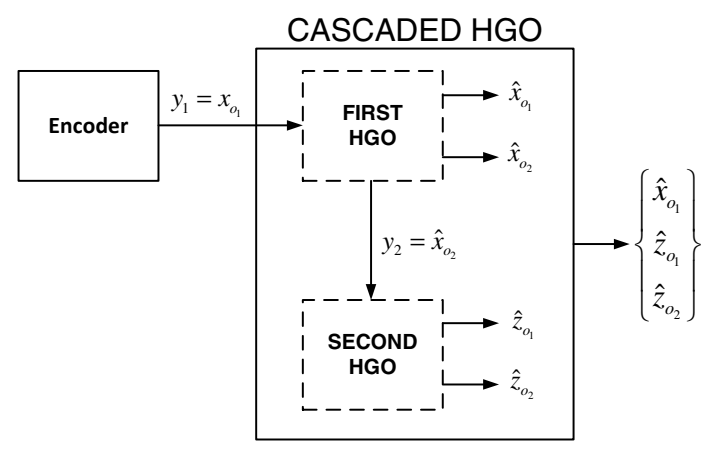

Fig. 2. Block diagram of Cascaded HGO Structure

The first HGO uses position measurements from an encoder to estimate position and velocity signals. The second HGO, on the other hand, utilizes estimated velocities by the first HGO to provide estimates of link accelerations. The dynamics of the first $\mathrm{HGO}$ is designed as:

$$
\begin{aligned}
& \dot{\hat{x}}_{o_{1}}=\hat{x}_{o_{2}}+L_{1}\left(y_{1}-\hat{x}_{o_{1}}\right) \\
& \dot{\hat{x}}_{o_{2}}=L_{2}\left(y_{1}-\hat{x}_{o_{1}}\right)
\end{aligned}
$$

where $\hat{x}_{o_{1}} \in \mathbb{R}^{n}$ and $\hat{x}_{o_{2}} \in \mathbb{R}^{n}$ are the estimated link positions and velocities, $\hat{x}_{o}(t)=\left[\begin{array}{ll}\hat{x}_{o_{1}} & \hat{x}_{o_{2}}\end{array}\right]^{T} \in \mathbb{R}^{2 n}$ denotes the observer state vector, $y_{1}=q_{m} \in \mathbb{R}^{n}$ is the encoder measurement given in (7), and the observer gains should be designed as:

$$
L_{1}=\frac{\beta_{1}}{\varepsilon_{1}}, \quad \text { and } \quad L_{2}=\frac{\beta_{2}}{\varepsilon_{1}^{2}}
$$

for some positive constants $\beta_{1}, \beta_{2} \in \mathbb{R}$, and $\varepsilon_{1} \ll 1$. Similarly, the dynamics of the second HGO is as follows:

$$
\begin{aligned}
& \dot{\hat{z}}_{o_{1}}=\hat{z}_{o_{2}}+L_{3}\left(y_{2}-\hat{z}_{o_{1}}\right) \\
& \dot{\hat{z}}_{o_{2}}=L_{4}\left(y_{2}-\hat{z}_{o_{1}}\right)
\end{aligned}
$$

where $\hat{z}_{o_{1}} \in \mathbb{R}^{n}$ and $\hat{z}_{o_{2}} \in \mathbb{R}^{n}$ are the estimated link velocities and accelerations, $\hat{z}_{o}(t)=\left[\begin{array}{ll}\hat{z}_{o_{1}} & \hat{z}_{o_{2}}\end{array}\right]^{T} \in \mathbb{R}^{2 n}$ represents the observer state vector, $y_{2}=\hat{x}_{o_{2}} \in \mathbb{R}^{n}$ is the estimated velocity by the first $\mathrm{HGO}$, and $L_{3}, L_{4}$ are the observer gains designed as follows:

$$
L_{3}=\frac{\beta_{3}}{\varepsilon_{2}}, \quad \text { and } \quad L_{4}=\frac{\beta_{4}}{\varepsilon_{2}^{2}}
$$

for some positive constants $\beta_{3}, \beta_{4} \in \mathbb{R}$, and $\varepsilon_{2} \ll 1$. Those observers are referred as high gain observers because larger observer gains, $L_{1}, L_{2}, L_{3}$, and $L_{4}$, are used in order to achieve zero estimation errors. High gain observers suffer from a peaking phenomenon due to sufficiently small $\varepsilon_{1}$ and $\varepsilon_{2}$. This phenomenon is handled by saturating the control input. The readers are referred to [15] for the details.

\section{Acceleration Feedback Based Learning CONTROLLER FOR ROBOTIC MANIPULATORS}

This section develops an acceleration based learning controller to achieve a global position tracking for n-rigid link robotic manipulators in (1).

\section{A. Controller Design}

The control objective is to design the torque control input signal, $\tau(t)$, such that the robot link positions will converge to desired trajectories despite parameter uncertainties in the dynamic model given in (1), i.e. $q(t) \Rightarrow q_{d}(t)$ as $t \Rightarrow \infty$. To quantify the control objective, the position tracking error, denoted by $e(t) \in \mathbb{R}^{n}$, is defined as follows:

$$
e=q_{d}-q
$$

where $q_{d}(t) \in \mathbb{R}^{n}$ is the desired link position.

\section{Assumption 1: Periodic and Bounded Desired Trajecto- ries}

The control objective is based on the assumption that $q(t)$, $\dot{q}(t)$ and $\ddot{q}(t)$ are measurable, and the desired link positions, velocities and accelerations are bounded, periodic functions of time that are defined as follows:

$$
q_{d}(t)=q_{d}(t-T), \quad \dot{q}_{d}(t)=\dot{q}_{d}(t-T),
$$

and

$$
\ddot{q}_{d}(t)=\ddot{q}_{d}(t-T)
$$

with a known period of $T$.

To facilitate the subsequent control development and stability analysis, we reduce the order of the robot dynamics given in (1) by defining a filtered tracking error variable, $s(t) \in \mathbb{R}^{n}$ as follows:

$$
s=\dot{e}+\Gamma_{1} e+\Gamma_{2} \int e d t
$$

where $\dot{e} \in \mathbb{R}^{n}$ is the link velocity error, i.e. $\dot{e} \triangleq \dot{q}_{d}-\dot{q}$, and $\Gamma_{1}, \Gamma_{2} \in \mathbb{R}^{n \times n}$ are constant, diagonal and positive-definite controller gain matrices. After taking the time derivative of (14) and multiplying the resulting expression by the inertia matrix, $M(q)$, the open loop error system is obtained as:

$$
M(q) \dot{s}=-C(q, \dot{q}) s+d+\psi-\tau
$$

where the auxiliary expression $d, \psi \in \mathbb{R}^{n}$ are defined as follows:

$$
d=M\left(q_{d}\right) \ddot{q}_{d}+C\left(q_{d}, \dot{q}_{d}\right) \dot{q}_{d}+G\left(q_{d}\right)+F_{v} \dot{q}_{d}+F_{s} \operatorname{sgn}\left(\dot{q}_{d}\right)
$$

and

$$
\begin{aligned}
\psi & =M(q)\left(\ddot{q}_{d}+\Gamma_{1} \dot{e}+\Gamma_{2} e\right)+G(q)+F_{v} \dot{q}+F_{s} \operatorname{sgn}(\dot{q})-d \\
& +C(q, \dot{q})\left(\ddot{q}_{d}+\Gamma_{1} e+\Gamma_{2} \int e d t\right)
\end{aligned}
$$

Since the real system parameters are not exactly known, the auxiliary signal, $d$, as a function of desired periodic 
trajectories, is an unknown periodic signal. In light of (2), (4) and (13), it follows that

$$
\left|d_{i}\right| \leq \eta_{i} \quad \text { for } \quad i=1,2, \ldots, n
$$

where $\eta_{i}=\left[\begin{array}{lll}\eta_{1} & \ldots & \eta_{n}\end{array}\right] \in \mathbb{R}^{n}$ is a vector of known, positive bounding constants.

By utilizing (2), (4), (12) and (14), and motivated by the result in [16], it is obtained that:

$$
\|\psi\| \leq \phi(\|v\|)\|v\|
$$

where the auxiliary signal $v(t) \in \mathbb{R}^{3 n}$ is defined as:

$$
v(t)=\left[\begin{array}{lll}
e^{T}(t) & s^{T}(t) & \ddot{e}^{T}(t)
\end{array}\right]^{T}
$$

and $\phi(\cdot) \in \mathbb{R}$ is a known and positive bounding function. On the basis of the structure of the open-loop error system in (15), the proposed control law is designed as follows:

$$
\tau=\Lambda s+\kappa \phi^{2}(\|v\|) s+\hat{d}
$$

where $\Lambda \in \mathbb{R}^{n \times n}$ is a constant, diagonal, positive-definite, controller gain matrix, $\kappa \in \mathbb{R}$ is a constant positive gain, and $\hat{d} \in \mathbb{R}^{n}$ is an estimate of $d$ in (16). Learning law, $\hat{d}$, is generated by modifying the learning feedforward term in [16] with acceleration feedback as:

$$
\hat{d}(t)=\operatorname{sat}_{\alpha}(\hat{d}(t-T))+K_{1} s+K_{2} \ddot{e}
$$

where $\ddot{e} \in \mathbb{R}^{n}$ is the link acceleration error, i.e. $\ddot{e} \triangleq \ddot{q}_{d}-\ddot{q}, K_{1}$ and $K_{2} \in \mathbb{R}^{n \times n}$ represent constant, diagonal, positive-definite, learning control matrices, respectively, and $\operatorname{sat}_{\alpha}(\cdot)$ denotes the saturation function and it is defined using the known, positive bounding constants given in (18):

$$
\operatorname{sat}_{\eta_{i}}\left(\varsigma_{i}\right)=\left\{\begin{array}{l}
\eta_{i}, \quad \varsigma_{i} \geq \eta_{i} \\
\varsigma_{i}, \quad-\alpha_{i}<\varsigma_{i}<\eta_{i} \\
-\eta_{i}, \quad \varsigma_{i} \leq-\eta_{i}
\end{array}\right.
$$

with $\forall \varsigma_{i} \in \mathbb{R}, i=1,2, . ., n$. In light of (23), the following inequality will be utilized in the subsequent stability analysis:

$$
\left(\varsigma_{1 i}-\varsigma_{2 i}\right)^{2} \geq\left(\operatorname{sat}_{\alpha_{i}}\left(\varsigma_{1 i}\right)-s a t_{\alpha_{i}}\left(\varsigma_{2 i}\right)\right)^{2}
$$

where $\forall\left|\varsigma_{1 i}\right| \leq \eta_{i}, \varsigma_{1 i} \in \mathbb{R}, i=1,2, \ldots, n$.

When (21) is substituted into (15), the closed-loop error system for $s(t)$ is obtained as:

$$
M \dot{s}=-C s-\Lambda s+\tilde{d}+\psi-\kappa \phi^{2}(\|v\|) s
$$

where $\tilde{d} \in \mathbb{R}^{n}$ is the learning estimation error:

$$
\tilde{d}=d-\hat{d}
$$

In light of (13), (16), (18) and (23), the following is derived:

$$
d(t)=\operatorname{sat}_{\alpha}(d(t))=\operatorname{sat}_{\alpha}(d(t-T))
$$

$\tilde{d}$ is obtained by substituting (22) and (27) into (26):

$$
\tilde{d}=s a t_{\alpha}(d(t-T))-s a t_{\alpha}(\hat{d}(t-T))-K_{1} s-K_{2} \ddot{e}
$$

\section{B. Closed-Loop Stability Analysis}

Theorem 1: The proposed controller developed in (21)-(22) can asymptotically drive the position error to zero, i.e.;

$$
\lim _{t \rightarrow \infty} e(t)=0
$$

where the controller gains $\Gamma_{1}, \Lambda, \kappa, K_{1}$ and $K_{2}$ given in (14), (21) and (22) are selected to satisfy the following sufficient condition

$$
\min \left(\left\|\Gamma_{1}\right\|,\left\|\Lambda+\frac{K_{1}^{T} K_{1}}{2}\right\|,\left\|\frac{K_{2}^{T} K_{2}}{2}\right\|\right)>\frac{1}{4 \kappa}
$$

where $\|\cdot\|$ is the 2-norm of a matrix, and there exists a firstorder differentiable, positive definite function $V_{5}(e, \dot{e}, \ddot{e}, t) \in \mathbb{R}$ such that

$$
\dot{V}_{1} \leq-e^{T} \Gamma_{1} e+s^{T} K_{1}^{T} K_{2} \ddot{e}+\tilde{d}^{T} K_{2} \ddot{e}+s^{T}\left(K_{1}-I\right) \tilde{d}
$$

where $I \in \mathbb{R}^{n \times n}$ is the identity matrix.

Proof: To prove the conclusion of Theorem 1, a Lyapunov function candidate, $V(t)$ is defined as

$$
\begin{gathered}
V=V_{1}+\frac{s^{T} M s}{2}+\frac{1}{2} \int_{t-T}^{t}\left(s a t_{\alpha} d(\phi)-s a t_{\alpha} \hat{d}(\phi)\right)^{T} \\
\left(\operatorname{sat}_{\alpha} d(\phi)-\operatorname{sat}_{\alpha} \hat{d}(\phi)\right) d \phi
\end{gathered}
$$

Taking the time derivative of (32), and using the Leibniz's Rule provided in Appendix B and the assumption given in (31) yields

$$
\begin{aligned}
\dot{V} \leq & -e^{T} \Gamma_{1} e+s^{T} K_{1}^{T} K_{2} \ddot{e}+\tilde{d}^{T} K_{2} \ddot{e}+s^{T}\left(K_{1}-I\right) \tilde{d}+s^{T} M \dot{s} \\
& +\frac{s^{T} \dot{M} s}{2}+\frac{1}{2}\left[( s a t _ { \alpha } d ( t ) - s a t _ { \alpha } \hat { d } ( t ) ) ^ { T } \left(s a t_{\alpha} d(t)\right.\right. \\
& \left.-s a t_{\alpha} \hat{d}(t)\right)-\left(s a t_{\alpha} d(t-T)-s a t_{\alpha} \hat{d}(t-T)\right)^{T} \\
& \left.\left(\operatorname{sat}_{\alpha} d(t-T)-s_{\alpha} \hat{d}(t-T)\right)\right]
\end{aligned}
$$

Using (3) and (25), the following is obtained:

$$
\begin{aligned}
\dot{V} \leq & -e^{T} \Gamma_{1} e+s^{T} K_{1}^{T} K_{2} \ddot{e}+\tilde{d}^{T} K_{2} \ddot{e}+s^{T} K_{1} \tilde{d}-s^{T} \Lambda s \\
& +s^{T} \psi-s^{T} \kappa \phi^{2} s+\frac{1}{2}\left\|s a t_{\alpha} d(t)-s a t_{\alpha} \hat{d}(t)\right\|^{2} \\
& -\frac{1}{2}\left(s a t_{\alpha} d(t-T)-s a t_{\alpha} \hat{d}(t-T)\right)^{T} \\
& \left(s_{\alpha} t_{\alpha} d(t-T)-s a t_{\alpha} \hat{d}(t-T)\right)
\end{aligned}
$$

The expression given in (34) can be rewritten based on (19) and (28) as follows:

$$
\begin{aligned}
\dot{V} \leq & -e^{T} \Gamma_{1} e+s^{T} K_{1}^{T} K_{2} \ddot{e}+\tilde{d}^{T} K_{2} \ddot{e}+s^{T} K_{1} \tilde{d}-s^{T} \Lambda s \\
& +\left[\phi\|v\|\|s\|-\kappa \phi^{2}\|s\|^{2}\right]+\frac{1}{2}\left\|s a t_{\alpha} d(t)-s a t_{\alpha} \hat{d}(t)\right\|^{2} \\
& -\frac{1}{2}\left[\left(\tilde{d}+K_{1} s+K_{2} \ddot{e}\right)^{T}\left(\tilde{d}+K_{1} s+K_{2} \ddot{e}\right)\right]
\end{aligned}
$$


By expanding the last line of (35), and performing cancellations, one obtains

$$
\begin{aligned}
\dot{V} \leq & -e^{T} \Gamma_{1} e-s^{T}\left(\Lambda+\frac{K_{1}^{T} K_{1}}{2}\right) s-\ddot{e}^{T} \frac{K_{2}^{T} K_{2}}{2} \ddot{e} \\
& +\frac{1}{2}\left[\left\|s a t_{\alpha} d(t)-s a t_{\alpha} \hat{d}(t)\right\|^{2}-\|d(t)-\hat{d}(t)\|^{2}\right] \\
& +\left[\phi\|v\|\|s\|-\kappa \phi^{2}\|s\|^{2}\right]
\end{aligned}
$$

By exploiting the property given in (24), completing the square on the bracketed term in the last line of (36), and using (20), (36) can be simplified as:

$$
\dot{V} \leq-\left[\min \left(\left\|\Gamma_{1}\right\|,\left\|\Lambda+\frac{K_{1}^{T} K_{1}}{2}\right\|,\left\|\frac{K_{2}^{T} K_{2}}{2}\right\|\right)-\frac{1}{4 \kappa}\right]\|v\|^{2}
$$

where $\|\cdot\|$ is the 2-norm of a matrix.

Signal Chasing: When (30) is satisfied, it follows that $V(t) \in$ $\mathscr{L}_{\infty}$ based on (32) and (37). Since the signals in $V(t)$ must remain bounded, it can be concluded that $s(t) \in \mathscr{L}_{\infty}$ and this implies $e(t) \in \mathscr{L}_{\infty}$ based on (14). It is also obtained from (37) that $v(t) \in \mathbb{L}_{2}$, and thus $v(t) \in \mathbb{L}_{\infty}$. The definition of $v(t)$ given in (20) implies $e(t), s(t), \ddot{e}(t) \in \mathbb{L}_{2} \cap \mathbb{L}_{\infty}$. Based on the fact that $s(t)$ is composed of $\dot{e}(t), e(t), \int e(t) d t$ as given in (14), and $s(t) \in \mathscr{L}_{2} \cap \mathscr{L}_{\infty}$, it follows that $\dot{e}(t) \in \mathscr{L}_{2} \cap \mathscr{L}_{\infty}$. Since $e(t), \dot{e}(t) \in \mathscr{L}_{\infty}$ and $e(t) \in \mathscr{L}_{2}$, Barbalat's Lemma [17] implies (29) in Theorem 1.

In light of (12) and (13), and using the boundedness of $e(t), \dot{e}(t), \ddot{e}(t)$, it follows that $q(t), \dot{q}(t), \ddot{q}(t) \in \mathscr{L}_{\infty}$. By exploiting the fact that the learning feedforward term given in (22) is composed of a saturation function, and $s(t), \ddot{e}(t) \in$ $\mathscr{L}_{\infty}$, it can be concluded that $\hat{d}(t) \in \mathscr{L}_{\infty}$. Finally, $\hat{d}(t), s(t) \in$ $\mathscr{L}_{\infty}$ implies $\tau(t) \in \mathscr{L}_{\infty}$ based on (21). Therefore all system signals remain bounded.

\section{Simulation Results}

The performance of the proposed learning controller is evaluated on a 2 DOF pan-tilt system. In order to construct a high fidelity simulation model, both nonlinear robot dynamics given in (5) and encoder model given by (7) are utilized. The desired trajectories which are presented in Figure 3 are generated based on the following periodic functions:

$$
\left[\begin{array}{l}
q_{d_{1}} \\
q_{d_{2}}
\end{array}\right]=\left[\begin{array}{l}
(3+0.1 \cos (t))(\sin (\sin (t)))\left(1+e^{-0.6 t^{3}}\right) \\
(1+0.1 \cos (t))(\sin (\sin (t)))\left(1+e^{-0.6 t^{3}}\right)
\end{array}\right]
$$

with a period of $T=6.28 \mathrm{sec}$ and the exponential term is used to provide a smooth-start to the system. The controller gains are tuned as follows:

$$
\begin{gathered}
\Gamma_{1}=\left[\begin{array}{cc}
20 & 0 \\
0 & 14
\end{array}\right], \Gamma_{2}=\left[\begin{array}{cc}
20 & 0 \\
0 & 20
\end{array}\right], \Lambda=\left[\begin{array}{cc}
40 & 0 \\
0 & 12
\end{array}\right] \\
K_{1}=\left[\begin{array}{cc}
30 & 0 \\
0 & 10
\end{array}\right], K_{2}=\left[\begin{array}{cc}
0.01 & 0 \\
0 & 0.01
\end{array}\right]
\end{gathered}
$$

Position and filtered errors reduce after each period of the desired trajectory as depicted in Figures 4 and 5. The
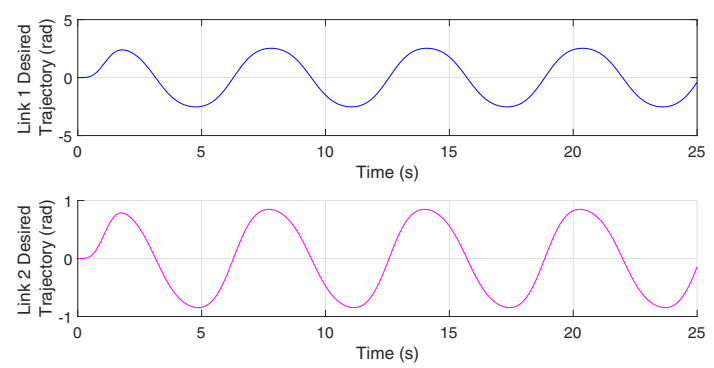

Fig. 3. Desired trajectories

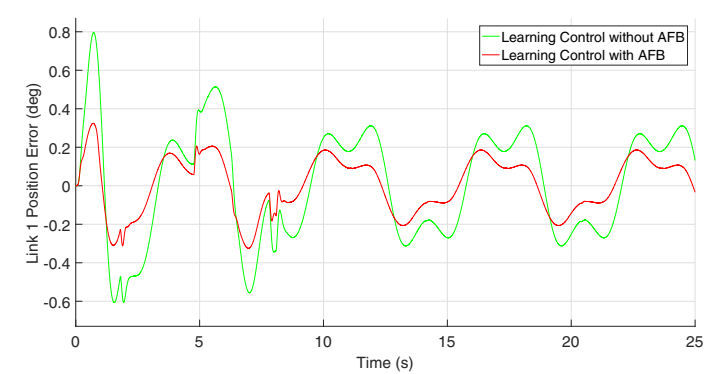

Fig. 4. Link 1 position error, $e_{1}(t)$

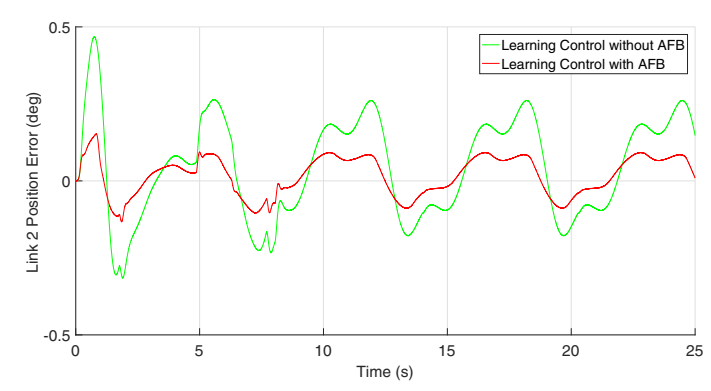

Fig. 5. Link 2 position error, $e_{2}(t)$

amplitude of oscillations are larger when the acceleration feedback is not utilized in the learning feedforward term.

Figures 6-9 depict the torque control inputs and the learning feedforward controls. Due to the desired periodic trajectories, control inputs oscillate to reject the unknown periodic disturbances. The proposed controller outperforms the learning controller without acceleration feedback as shown in Tables I and II.

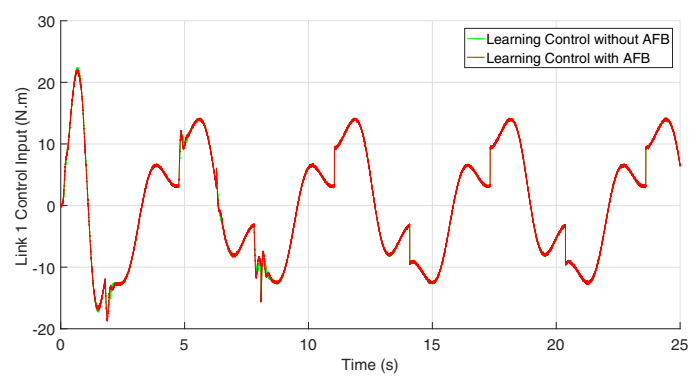

Fig. 6. Link 1 control input, $\tau_{1}(t)$ 


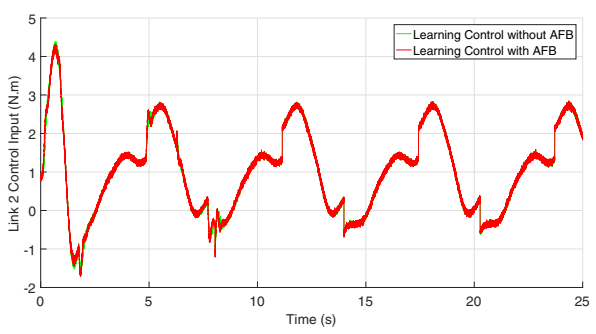

Fig. 7. Link 2 control input, $\tau_{2}(t)$

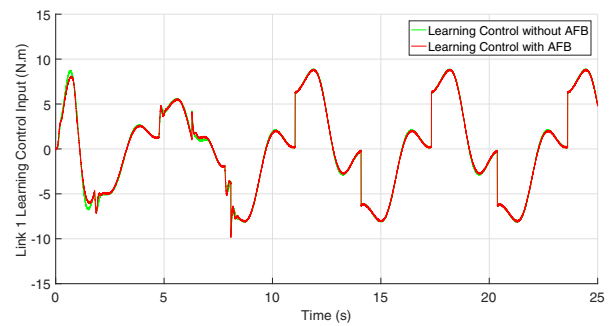

Fig. 8. Link 1 learning feedforward control, $d_{1}(t)$

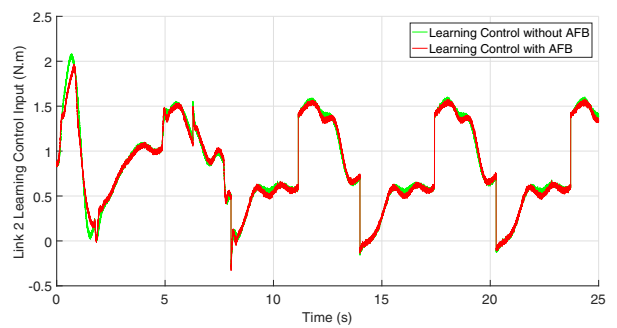

Fig. 9. Link 2 learning feedforward control, $d_{2}(t)$

TABLE I

LINK 1 PERFORMANCE SPECIFICATION

\begin{tabular}{ccc}
\hline $\begin{array}{c}\text { Performance } \\
\text { Criteria }\end{array}$ & $\begin{array}{c}\text { Proposed } \\
\text { Controller }\end{array}$ & $\begin{array}{c}\text { Learning Controller } \\
\text { without AFB }\end{array}$ \\
\hline Worst Case Position Error (deg) & 0.3244 & 0.7959 \\
RMS Position Error (deg) & 0.1450 & 0.2910 \\
RMS Control Input (N.m) & 9.1981 & 9.2276 \\
\hline
\end{tabular}

TABLE II

LINK 2 PERFORMANCE SPECIFICATION

\begin{tabular}{ccc}
\hline $\begin{array}{c}\text { Performance } \\
\text { Criteria }\end{array}$ & $\begin{array}{c}\text { Proposed } \\
\text { Controller }\end{array}$ & $\begin{array}{c}\text { Learning Controller } \\
\text { without AFB }\end{array}$ \\
\hline Worst Case Error (deg) & 0.1527 & 0.4683 \\
RMS Error (deg) & 0.0656 & 0.1689 \\
RMS Control Input (N.m) & 1.5248 & 1.5283 \\
\hline
\end{tabular}

\section{CONCLUSIONS}

We have now developed a new acceleration based learning controller for the periodic trajectory tracking of robotic manipulators. The robustness of the system against unknown periodic disturbances is improved with the use of acceleration feedback in the learning feedforward term. A new cascaded high gain observer is utilized to estimate position, velocity and acceleration signals from noisy encoder measurements. Estimates produced by the observer are used as feedback signals in the proposed learning controller. By a closed loop stability analysis, it is shown that all system signals remain bounded and global asymptotic position tracking is ensured. The performance of the proposed learning controller is tested on a high fidelity simulation model of a 2 DOF pan-tilt platform. Results show that smaller position errors are achieved when acceleration feedback is utilized in the learning controller.

\section{REFERENCES}

[1] S. Arimoto, S. Kawamura, F. Miyazaki, "Bettering operation of robots by learning," Journal of Field Robotics, Vol. 1, No. 2, pp. 123-140, 1984.

[2] S. Hara, Y. Yamamoto, T. Omata, M. Nakano, "Repetitive control system: A new type servo system for periodic exogenous signals," IEEE Transactions on Automatic Control, Vol. 33, No. 7, pp. 659668, 1988.

[3] M. Hehn, R. D'Andrea, "A frequency domain iterative learning algorithm for high-performance, periodic quadrocopter maneuvers," Mechatronics, Vol. 24, No. 8, pp. 954-965, 2014.

[4] X. Li, Q. Ren, J. Xu, "Precise Speed Tracking Control of a Robotic Fish Via Iterative Learning Control," IEEE Transactions on Industrial Electronics, Vol. 63, No.4, pp. 2221-2228, 2016.

[5] D. H. Owens, E. Rogers, K. Galkowski, "Control theory and applications for repetitive processes," Advances in Control, Vol. 2, No. 3, pp. 327-333, 1999.

[6] W. Messner, R. Horowitz, W. W. Kao, M. Boals, "A New Adaptive Learning Rule," IEEE Transactions on Automatic Control, Vol. 36, No. 2, pp. 188-197, 1991

[7] W. E. Dixon, E. Zergeroglu, D. M. Dawson, B. T. Costic, "Repetitive learning control: A lyapunov-based approach," IEEE Transactions on Systems, Man, and Cybernetics, Vol. 32, No. 4, pp. 538-545, 2002.

[8] W. J. Cao, and J. X. Xu, "Robust and almost perfect periodic tracking of nonlinear systems using repetitive VSC," American Control Conference, pp. 3830-3835, 2001.

[9] C. Lin, and Y. Zeng, "Synergetic repetitive controla plug-in hybrid control method for precision motion control," The International Journal of Advanced Manufacturing Technology, Vol. 87, No. 1-4, pp. 19-24, 2016.

[10] L. Wang, C. Freeman, S. Chai, E. Rogers, "Repetitive-predictive control system with constraints: From design to implementation," Journal of Process Control, Vol. 23, No. 7, pp. 956-967, 2013.

[11] L. Wang, C. Freeman, E. Rogers, "Predictive iterative learning control with experimental validation," Control Engineering Practice, Vol. 53, pp. 24-24, 2016.

[12] M. W. Spong, S. Hutchinson, M. Vidyasagar, Robot Modeling and Control, John Wiley and Sons, USA, 2006.

[13] G. Tao, X. Ma, "Backlash compensation for multivariable nonlinear systems with actuator dynamics," IEEE Conference on Decision and Control, pp. 3382-3387, 1999.

[14] S. Evren and M. nel, "High Precision Stabilization of Pan-Tilt Systems Using Reliable Angular Acceleration Feedback from a MasterSlave Kalman Filter," Journal of Intelligent and Robotic Systems, DOI:10.1007/s10846-017-0522-9, 2017.

[15] H. K. Khalil, L. Praly, "High-gain observers in nonlinear feedback control," International Journal of Robust and Nonlinear Control, Vol. 24, No. 6, pp. 993-1015, 2014.

[16] W. E. Dixon, A. Behal, D. M. Dawson, S. P. Nagarkatti, Nonlinear control of engineering systems: A lyapunov-based approach, Birkhauser, 2003.

[17] H. K. Khalil, Nonlinear Systems, Prentice-Hall, New Jersey, 2002. 